\title{
Trauma exposure and post-traumatic stress disorder in a cohort of pregnant Peruvian women
}

\section{( 遇 Article in press ? )}

- Levey, E.J. ${ }^{a b c}$ Email Author,

- Gelaye, B. ${ }^{d}$,

- Koenen, K. ${ }^{\text {abd }}$,

- Zhong, Q.-Y.d,

- Basu, A. abd,

- Rondon, M.B. ${ }^{\mathrm{e}}$,

- Sanchez, S. ${ }^{f}$,

- Henderson, D.C. ${ }^{\text {gh }}$,

- Williams, M.A. ${ }^{d}$

- View Correspondence (jump link)

- $\quad$ aThe Chester M. Pierce, MD Division of Global Psychiatry, Massachusetts General Hospital, Boston, MA, United States

- $\quad$ bHarvard Medical School, Boston, MA, United States

- Institute for Juvenile Research, University of Illinois College of Medicine, Chicago, IL, United States

- $\quad$ Harvard T.H. Chan School of Public Health, Boston, MA, United States

- Universidad Peruana Cayetano Heredia, Lima, Peru

- $\quad$ Unniversidad Peruana de Ciencias Aplicadas, Lima, Peru

- $\quad{ }^{9}$ Department of Psychiatry, Boston Medical Center, Boston, MA, United States

- $\quad$ hBoston University School of Medicine, Boston, MA, United States

Hide additional affiliations_

\section{Abstract}

Women have a higher prevalence of post-traumatic stress disorder (PTSD) than men, with a peak during the reproductive years. PTSD during pregnancy adversely impacts maternal and infant health outcomes. The objectives of this study were to estimate the prevalence of antepartum PTSD symptoms in a population of pregnant Peruvian women and to examine the impact of number of traumatic events and type of trauma experienced. The Traumatic Events Questionnaire was used to collect data about traumatic exposures. The Post-traumatic Stress Disorder Checklist-Civilian Version (PCL-C) was used to assess PTSD. Multivariable logistic regression procedures were used to estimate adjusted odds ratios $(\mathrm{aOR})$ and 95\% confidence intervals $(95 \% \mathrm{Cl})$. Three thousand three hundred seventy-two pregnant women were interviewed. Of the 2920 who reported 
experiencing one or more traumatic events, $41.8 \%$ met criteria for PTSD (PCL-C score $\geq 26$ ). A quarter of participants had experienced four or more traumas, and $60.5 \%$ of those women had PTSD. Interpersonal trauma was most strongly associated with PTSD (aOR, 3.20; 95\% Cl, 2.74-3.74), followed by unspeakable trauma (aOR, 2.87; 95\% Cl, 2.35-3.50), and structural trauma (aOR, $1.39 ; 95 \% \mathrm{Cl}$, 1.15-1.67). These findings indicate the high prevalence of PTSD during pregnancy in the Peruvian population, which is relevant to other countries suffering from terrorism, war, or high rates of violence. This underscores the importance of screening for PTSD in pregnancy. (C) 2017 Springer-Verlag GmbH Austria

Author keywords

- Peru

- Pregnancy

- PTSD

- TEQ

- Trauma

- ISSN: 14341816

- CODEN: AWMHF

- Source Type: Journal

- Original language: English

- DOI: $10.1007 / \mathrm{s} 00737-017-0776-z$

- Document Type: Article in Press

- Publisher: Springer-Verlag Wien 